\title{
Source Regions of Coronal Mass Ejections
}

\author{
K. P. Dere \\ Naval Research Laboratory, Code 7660, Washington, DC 20375, USA \\ P. Subramanian \\ National Center for Radio Astrophysics, Tata Institute of Fundamental \\ Research, Pune - 411007, India
}

\begin{abstract}
Observations of the solar corona with the LASCO and EIT instruments on SOHO provide an unprecedented opportunity to study coronal mass ejections (CMEs) from their initiation through their evolution out to $30 \mathrm{R}_{\odot}$. The objective of this study is to gain an understanding of the source regions from which the CMEs emanate. To this end, we have developed a list of 32 CMEs whose source regions are located on the solar disk and are well observed in EIT $195 \AA$ data during the solar minimum phase of January 1996-May 1998. We compare the EIT source regions with photospheric magnetograms from the Michelson Doppler Imager (MDI) instrument on SOHO and the NSO/Kitt Peak Observatory and also with $\mathrm{H} \alpha$ data from various sources. The overall results of our study show that $59 \%$ of the CME related transients observed in EIT 195 $\AA$ images are associated with active regions without prominences, $22 \%$ are associated with eruptions of prominences embedded in active regions and $19 \%$ are associated with eruptions of quiescent prominences. We describe 3 especially well observed events, one from each of these 3 categories. These case studies suggest that active region CMEs are associated with active regions with lifetimes between 11-80 days. They are also often associated with small scale emerging or cancelling flux over timescales of 6-7 hours. CMEs associated with active region prominence eruptions, on the other hand, are typically associated with old active regions with lifetimes $\sim 6-7$ months.
\end{abstract}

\section{Introduction}

In this paper we examine the solar source regions of coronal mass ejections (CMEs) which have been observed in the solar corona with the LASCO coronagraphs on SOHO. A primary goal has been to understand the magnetic configuration of the coronal regions that are involved in the initiation of the CME. Consequently, the primary data sources that have been examined include the EIT $195 \AA$ images, the MDI photospheric magnetograms and the NSO/Kitt Peak synoptic magnetic maps. Since the MDI magneograms are most useful when examining events toward disk center, we have limited our study to event that are away from the extreme solar limb. In addition, we have confined our study to 
times when relatively high cadence $(12-18 \mathrm{~m})$ and full resolution $(2.5$ arc-sec per pixel) EIT images were obtained. The photons detected by the EIT $195 \AA$ channel are primarily due to Fe XII which is formed at a temperature around $1.4 \times 10^{6} \mathrm{~K}$. We have limited our data set to the time period from the beginning of high data-rate observations with EIT in April 1997 through the period when SOHO was temporarily lost in June 1998. We selected 32 CMEs that met our criteria in this time period for detailed analysis.

We have concentrated on several aspects of the CME observations that we have selected. We have attempted to understand the structure of the corona preceding the CME and the evolution of the coronal structures at the onset of the CME and the propagation of the CME through the lower corona. We have examined the NSO/Kitt Peak synoptic magnetograms to understand the long term evolution of the photospheric magnetic fields associated with the CME and the MDI magnetograms to understand the short-term, small-scale evolution of the photospheric magnetic fields prior to the CME.

Several studies have been carried out in the past on the association between CMEs with other kinds of solar activity such as flares and disappearing prominences. Munro et al. (1979) used CME observations from Skylab data during 1973-1974 near solar minimum in conjunction with reports of associated activity from the Solar Geophysical Data archives. They found that $78 \%$ of the CMEs observed were associated with other forms of solar activity. Of these, they found that $40 \%$ of the CMEs observed are associated with flares, while more than $70 \%$ of CMEs are associated with eruptive prominences (with and without flares). Webb and Hundhausen (1987) examined SMM data during 1980 near solar maximum and found that $66 \%$ of the CMEs observed were associated with other forms of solar activity. Of these, they found that $68 \%$ of the observed CMEs were associated with erupting prominences, $37 \%$ with $\mathrm{H} \alpha$ flares and $47 \%$ with X-ray events. St. Cyr and Webb (1991) analyzed SMM data from 1984-1986, during the declining phase of the solar cycle. They find that slightly less than half of the CMEs have associations with other forms of solar activity. Of these, they found that $76 \%$ were associated with erupting prominences, $26 \%$ with $\mathrm{H} \alpha$ flares and $74 \%$ with X-ray events.

With regard to the relationship between CMEs and the photospheric magnetic field Feynman \& Martin (1995) find a strong correlation between newly emerging magnetic field and eruptions of quiescent filament, which they take to be proxies for CMEs. Luhmann et al. (1998) employ potential field extrapolations to determine the large scale coronal field from the photospheric magnetic field. Their results suggest that although the changes in the small scale photospheric magnetic field before and after a CME might be indiscernible, CMEs could correspond to the opening up of large scale coronal field lines. Lara, Gopalswamy, \& DeForest (2000) examined 8 eruptive events associated with active regions during 1997 and 1998. They find that the total magnetic flux over an active region did not show significant changes. However, they found significant changes in the flux over small subregions of the overall active region associated with the CMEs. They also found that the changes in flux occur over timescales of several hours to days. A demonstration of a causal relationship between photospheric flux changes and CME initiation remains to be provided. 
Photospheric magnetic field configurations are the boundary conditions for determining the coronal magnetic field which is disrupted during a CME. Canfield, Hudson and McKenzie (1999) have suggested that CME source regions often correspond to structures resembling sigmoids in YOHKOH soft X-ray images, that presumably trace highly stressed coronal magnetic field configurations. Falconer (2000) suggests quantitative measures of the global nonpotentiality of active regions that might determine whether or not they will produce CMEs.

The issue of CME initiation is thus an issue of considerable contemporary interest. The combination of the highly sensitive white light images provided by the LASCO coronograph, on-disk EIT images and 1.5 hour cadence (MDI) photospheric magnetograms provide a unique opportunity to investigate the complex question of CME initiation. This kind of comprehensive data set was not available when previous studies, described above, of the associations between CMEs and disk phenomena were made. We address the question of CME initiation by analyzing the source regions of CMEs observed on the solar disk in EIT $195 \AA$ images during the solar minimum period of January 1996-June 1998.

\section{Overall Results}

We have identified the source regions on the CMEs in our set of 32 events from their signature in the EIT $195 \AA$ spectroheliograms. We have determined that all of the events originated from either an active region or a quiescent prominence. During this phase of the solar cycle, active regions are usually well isolated and these identifications tend to be straightforward. On-disk prominence eruptions are occasionally clearly evident in EIT $195 \AA$ images. We have also examined $\mathrm{H} \alpha$ data from various sources such as the Big Bear Solar Observatory, Observatory of Paris at Meudon, Holloman Air Force Base and the Hiraiso Solar Terrestrial Research Center to determine if CME-related transients in EIT $195 \AA$ images are related to pre-existing prominences. The $\mathrm{H} \alpha$ data available from these sources often have rather poor cadence in comparison to the other data we use; images are typically taken once or twice a day. We are therefore fairly liberal in assigning associations with prominence disappearances. If, for instance, we observe a prominence in $\mathrm{H} \alpha$ data near the source region of the CME on the day before the CME, and if it has partially or completely disappeared in an image taken after the CME has occurred, we conclude that the CME was associated with a prominence eruption. We have carried out this procedure for all the 32 events in our catalog. This exercise allows us to place the source regions of CMEs into the following broad categories:

1. Active regions (AR category). There is no evidence of prominences embedded in these active regions.

2. Active regions that contain a filament ( $\mathrm{AR}+\mathrm{P}$ category). The prominences in this category are typically embedded in old, decaying active regions. Such regions might not necessarily be assigned a NOAA active region number.

3. Quiescent prominences ( $\mathrm{P}$ category). The prominences in this category occur in quiet regions where the magnetic fields are relatively weak. 


\section{AR}

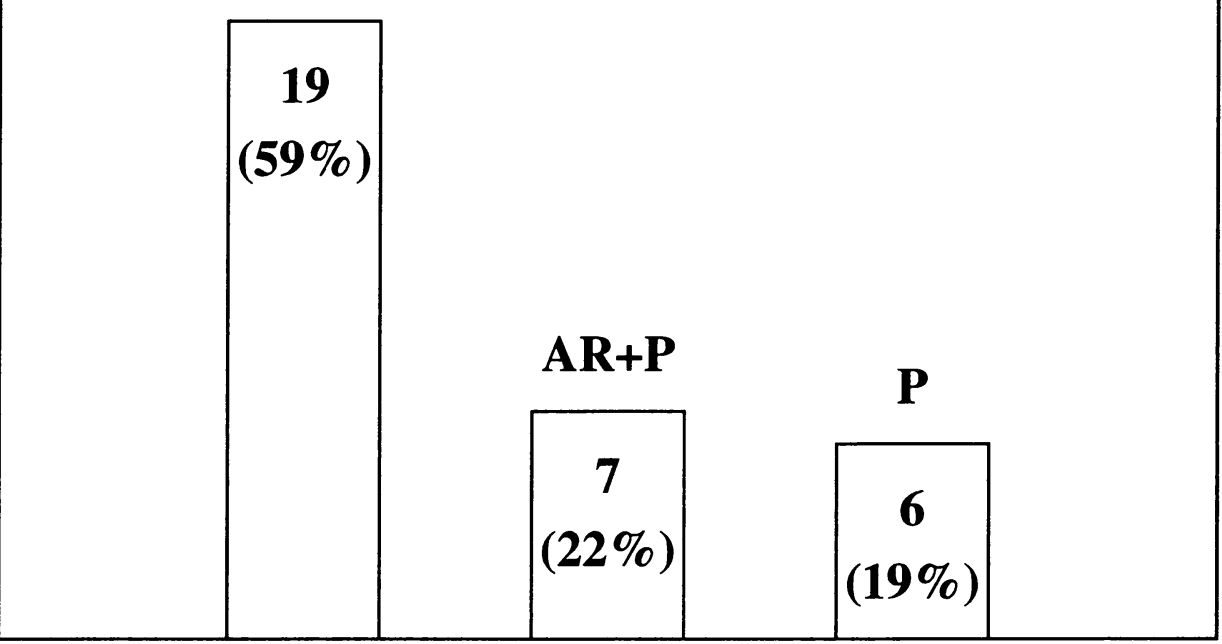

Figure 1. Histogram of CME source region frequency

These three categories account for the source regions of all of the CMEs in our selection of events. The association of CME source regions with active regions (events in category 1 ) is made by visual inspection of EIT $195 \AA$ images and synoptic magnetograms. The association with erupting prominences, on the other hand, is made by inspecting EIT $195 \AA$ images as well as $\mathrm{H} \alpha$ data from various sources. As summarized in Figure 2, we find that $59 \%$ of the cataloged events fall in the AR category, while $22 \%$ are in the AR+P category and $19 \%$ are in the $\mathrm{P}$ category.

Feynman and Martin (1995) find that quiescent filament eruptions (which they take to be proxies for CMEs) are strongly correlated with the emergence of new magnetic flux over several days preceding the eruption. Our findings in this respect are different. As shown in Figure 1, 7 of the 32 events on our list are in the AR+P category, which was not included in the study by Feynman and Martin (1995), while 6 of the 32 events on our list are in the P category. The CMEs in the $\mathrm{P}$ category arise from eruptions of prominences from quiet regions. There is no evidence for significant large-scale magnetic flux emergence for any of the 6 quiescent filament eruptions that we observed. Nevertheless, we have observed quiescent filament eruptions that were associated with newly emerged magnetic flux, for example, the west limb CME on 1996 July 10. This event was not included in our study because of the lack of high cadence EIT data. 


\section{Case Studies}

Sorting the various CME source regions into 3 categories provides a useful overall description of the source regions of CMEs. Nevertheless, such a broad characterization covers over much of the complex nature of the CME source regions and the CME initiation. We have therefore selected 3 especially well observed events from the list of 32 events for closer examination. This subset includes one event from each of the three categories: active region (AR), active region with prominence $(A R+P)$, and quiescent prominence $(P)$. The principal criterion used in the selection of this subset of 3 events was the availability of high cadence EIT $195 \AA$ images. For these events, we have examined the EIT 195 $\AA$ images in detail and made detailed comparisons of the EIT $195 \AA$ images with full disk photospheric magnetograms from the MDI instrument in order to determine the spatial and temporal relationship between the photospheric magnetic field evolution and the CME-related transient in EIT $195 \AA$ images. We have studied the NSO/Kitt Peak synoptic magnetic maps to determine the long-term evolution of the photospheric fields. We have also examined X-ray light curves from GOES data to determine if there are x-ray flares associated with each of these events.

\subsection{The CME of 01 April 1997 (AR)}

This is an example of a CME that originates in an active region (AR), AR 8026 observed during Carrington rotation 1921. The MDI magnetogram and EIT $195 \AA$ spectroheliogram are show in Figure 2 . The active region was absent on the previous rotation, and emerged on the back side of the sun after 14 March 1997. Since it is an isolated active region, loops associated with this AR can be discerned from LASCO C1 images as early as 22 March 1997, well before the AR reaches the East limb. The AR is first apparent in NSO Kitt Peak magnetograms at (S23E78) during rotation 1921 on 27 March 1997. The CME of 01 April 1997 occurs when the AR is at S24E20. Following the CME, the active region weakens and disperses, but retains its identity well into rotation 1922 . It was last seen near the west limb on 13 May 1997 during rotation 1922 in MDI magnetograms and does not reappear at the east limb on the subsequent rotation. In summary, the active region, with which the CME of April 11997 is associated, appeared between 14 March 1997 and 22 March 1997 and disappeared between 13 May 1997 and 26 May 1997. The lifetime of the active region is between 53 and 75 days. The CME occurred early in the life of the active region.

AR 8026 consists of two compact regions of opposite magnetic polarities, with a parasitic negative polarity embedded in the positive polarity region (Figure 2). On 01 April 1997, the sunspot corresponding to AR 8026 is assigned a $\beta$ classification. On 1 April 1997, the MDI magnetograms show a fair amount of small-scale flux emergence and cancellation, particularly along the neutral line and the western edge of the active region. The parasitic negative polarity region is evident in the MDI magnetogram images from as early as 23:59 UT on 31 March 1997. The parasitic negative polarity evolves from being embedded in the positive polarity region at 08:03 UT to forming a lane between two segments of the positive polarity region by 14:27 UT. This trend continues, and we find that the negative parasitic polarity lane has elongated further by $17: 39$ UT. 

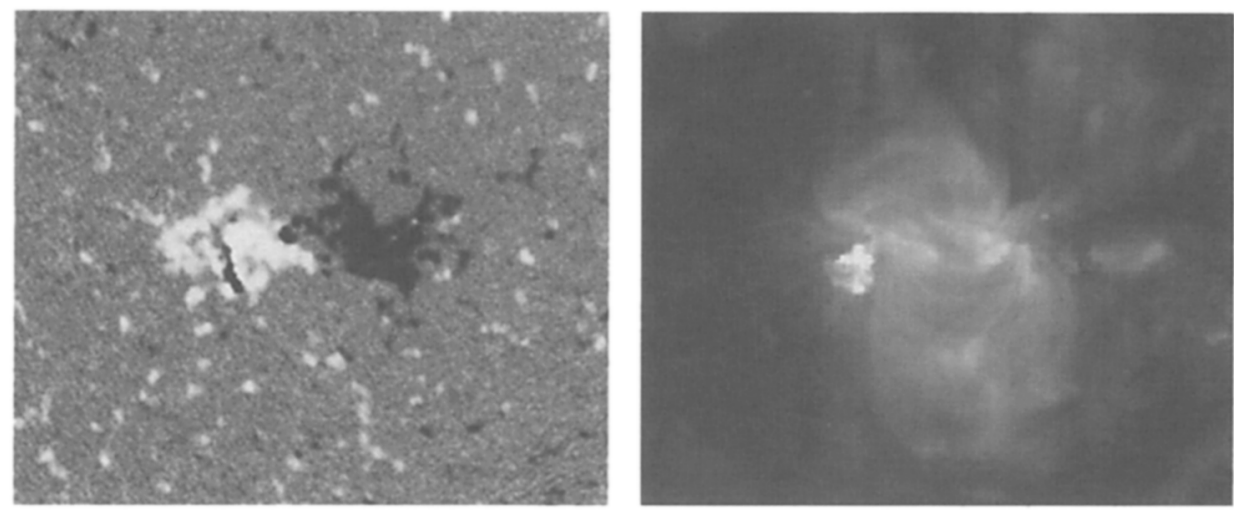

Figure 2. MDI/SOHO magnetogram of AR 8026 on 1997 April 1 at 1427 UT. Right, the co-aligned EIT $195 \AA$ spectroheliogram at 1346 UT.

Near the time of the CME, the first sign of activity in EIT $195 \AA$ images is a brightening at 13:25 UT apparently at the top of the arch connecting the dominant polarities of AR 8026. This is followed by a brightening at 13:46 UT near the eastern leg of the bipolar arch, around the location of the parasitic polarity. The brightening near the parasitic polarity is clearly evident in the straight EIT $195 \AA$ image at 14:00 UT. This sequence of events is accompanied by an M1.9 X-ray flare that starts at 13:25 UT, peaks at 13:48 UT and ends at 14:01 UT. An EIT wave is observed to emanate from the vicinity of the active region at 14:00 UT (Fig. 3). The northwestern front of this disturbance is the only one that can be clearly identified as an outwardly propagating feature in successive images. It starts out with a velocity of $\sim 100 \mathrm{~km} \mathrm{~s}^{-1}$ and propagates with an acceleration of $\sim 70 \mathrm{~ms}^{-2}$. By the time it reaches the northwest edge of the disk, it has attained a speed of $\sim 300 \mathrm{~km} \mathrm{~s}^{-1}$. A stationary dimming region is observed to the southwest of the active region. It is manifested as a deepened darkening at approximately the same location in the running difference EIT $195 \AA$ image at 14:18 UT. We also observe a stationary transient dimming at 14:18 UT to the southeast of the active region. We have checked constant base difference EIT $195 \AA$ images to verify that these features are not artifacts of the running difference technique. In summary, the activity observed in EIT $195 \AA$ images consists of a flare(s), a bright EIT wave propagating towards the northwest and dimming regions to the southeast and southwest of the active region.

The LASCO C2 observations of the CME are shown in Figure 4. These running difference LASCO C2 images show a wide, bright mound on the east limb appearing simultaneously with a small bright ejection on the west limb at 15:18 UT. The wide front on the east limb travels outward at a speed of 296 $\mathrm{km} \mathrm{s}^{-1}$. The appearance of this CME is considerably different from those that are typically ejected from the limb but is consistent with a CME ejected from the solar disk in the general direction of SOHO. The simultaneous ejection from the two limbs suggests that this CME could be a helical CME propagating towards 

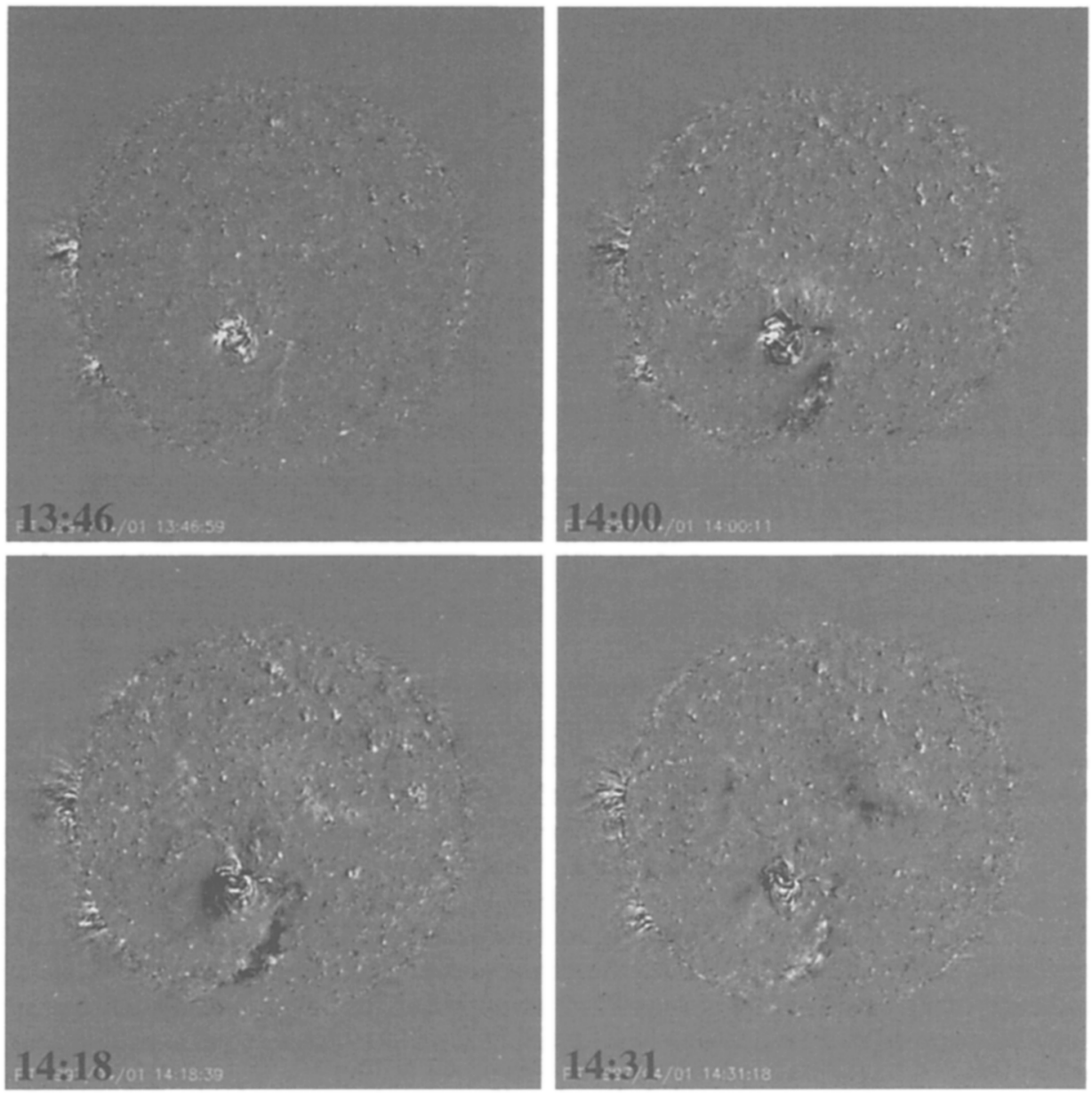

Figure 3. EIT $195 \AA$ running difference images of the 1 April 1997 CME.

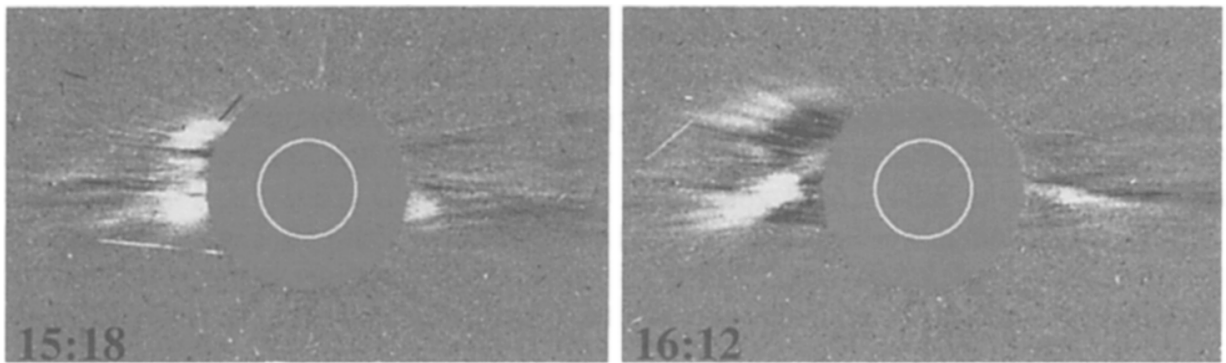

Figure 4. LASCO C2 running difference images of the 1 April 1997 CME. 
the earth (Dere et al. 1999). It is worth noting that the EIT wave propagates to the northwest, whereas the CME observed in LASCO C2 images is concentrated along the equator, with the brightest feature on the east limb. Consequently, the propagating front in EIT $195 \AA$ data is probably not directly related to the white light CME. However, the visibility of the EIT wave on the disk could be non-uniform, and the northwestern feature could be the most evident one. Furthermore, CMEs originating near the limb that start out propagating at relatively large angles to the equator eventually tend to follow the large-scale dipole field and bend towards the equator as they propagate out.

\subsection{The CME of 21 October $1997(\mathrm{AR}+\mathrm{P})$}

The source region of this event is classified in the AR+P category. It is an old, decaying active region complex that includes AR 8097 and the magnetic fields to its northeast (Figure 5). Synoptic magnetograms from the NSO Kitt Peak reveal that this active region complex emerges during Carrington rotation 1926. It then undergoes considerable evolution, but does not disappear until Carrington rotation 1932. The lifetime of the overall complex is thus $\sim 6$ months, and this CME occurs $\sim 3$ months after the complex first appeared. The emergence of AR 8097 represents the emergence of new flux into the original active region complex. AR 8097 was not present at the west limb during rotation 1927 on 02 October 1997. It grew on the backside of the Sun and rotated into view at the East limb in Kitt Peak NSO magnetogram data on 16 October 1997 (Carrington rotation 1928). There was evidence for loops associated with this active region in EIT $195 \AA$ images when this active region was behind the east limb, on 14 October 1997. We therefore conclude that AR 8097 emerged between 02 October 1997 and 14 October 1997. Synoptic magnetograms from the NSO Kitt Peak reveal that this active region decayed slowly and disappeared between 13 January 1998 and 12 February 1998. There is evidence for small-scale flux emergence, cancellation and decay in the photospheric magnetic field, but the relation of these changes to the activity in EIT $195 \AA$ images is not clear. In summary, the CME of 21 October 1997 occured during the rising phase of new flux injected into an old, decaying active region complex.

The activity observed in the EIT $195 \AA$ images includes a bright EIT wave accompanied by propagating density depletions, a prominence ejection, the ejection of bright material to the southeast and flare-associated brightenings. The EIT $195 \AA$ images available around the time of this event suggest that an EIT wave is initiated from AR 8097 between 16:18 UT and 17:34 UT. The bright wave is observed to spread in a semi-isotropic manner, propagating preferentially towards the south. The most pronounced wave-associated darkenings (which could be interpreted as density depletions) are observed towards the north and south of the active region. The propagating depletion wave is also accompanied by material ejection that is manifested as the bright N-S feature at the southwestern boundary of the dark region. This event is accompanied by a C3.3 flare that starts at 17:00 UT, peaks at 17:54 UT and ends at 18:16 UT. At 17:34 UT we observe a bright linear feature near the magnetic neutral line that we interpret as the prominence activation (Fig. 6). Later, at 17:45 UT, we observe a doubleribbon flare-associated brightening on either side of the prominence and at the 

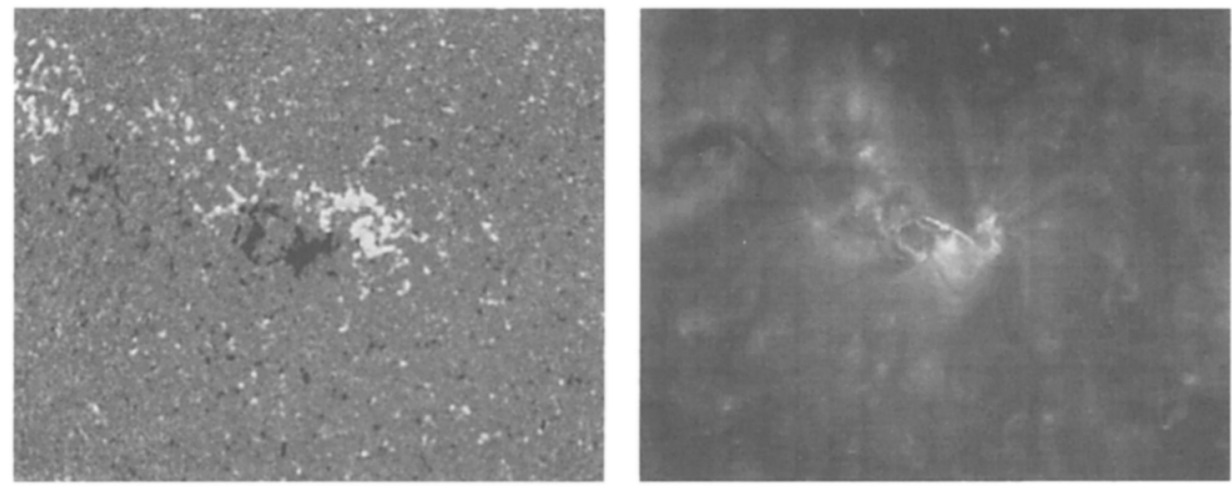

Figure 5. Left, MDI magnetogram of AR 8026 at 1739 UT. Right, EIT $195 \AA$ spectroheliogram at 1734 UT with bright eruptive filament evident.

footpoints of the post-flare loops which are later seen in EIT $195 \AA$ images at 18:12 UT.

The $\mathrm{H} \alpha 6563 \AA$ image from the Big Bear Solar Observatory taken at 15:26 UT on 21 October 1997 reveals an embedded filament in the active region. The corresponding image taken at 15:45 UT on 22 October 1997 shows that the embedded filament has largely disappeared. It is therefore quite likely that this $\mathrm{CME}$ is associated with the eruption of a part of this embedded prominence. Consequently, we have place this CME in the active region with prominence $(\mathrm{AR}+\mathrm{P})$ category.

This sequence of events is associated with a halo CME at 18:18 UT in LASCO C2 data. The CME front is brightest on the east limb, although a faint front extends all over the northen hemisphere. The southwestern front of the halo is somewhat delayed with respect to the other fronts. The leading edge on the east limb travels outward at a speed of $465 \mathrm{~km} \mathrm{~s}^{-1}$, with no appreciable acceleration.

\subsection{The CME of 19 May 1998 (P)}

The source of the 19 May $1998 \mathrm{CME}$ is a quiescent eruptive prominence which places this event in the ' $\mathrm{P}$ ' category. The prominence is formed along the neutral line of the relatively weak, extended magnetic fields to the west of AR 8222 (Fig. 7 ). These fields appear to be the dispersed remnants of AR 8203 which emerged during the previous rotation (rotation 1935). We studied the active region and the weaker magnetic fields to its west for a period of 5 days before the CME. We found no evidence for significant large-scale emerging or cancelling flux near the location of the prominence over this timescale. There are no significant small-scale changes in magnetic flux over timescales of a few hours either.

The EIT $195 \AA$ image (Fig. 7) shows the prominence eruption which started at 09:34 UT and is well in progress by 09:56 UT. The prominence eruption travels out behind the CME front and is well visible in the LASCO C2 data shown in Fig. 8. There were no flares associated with this CME. 

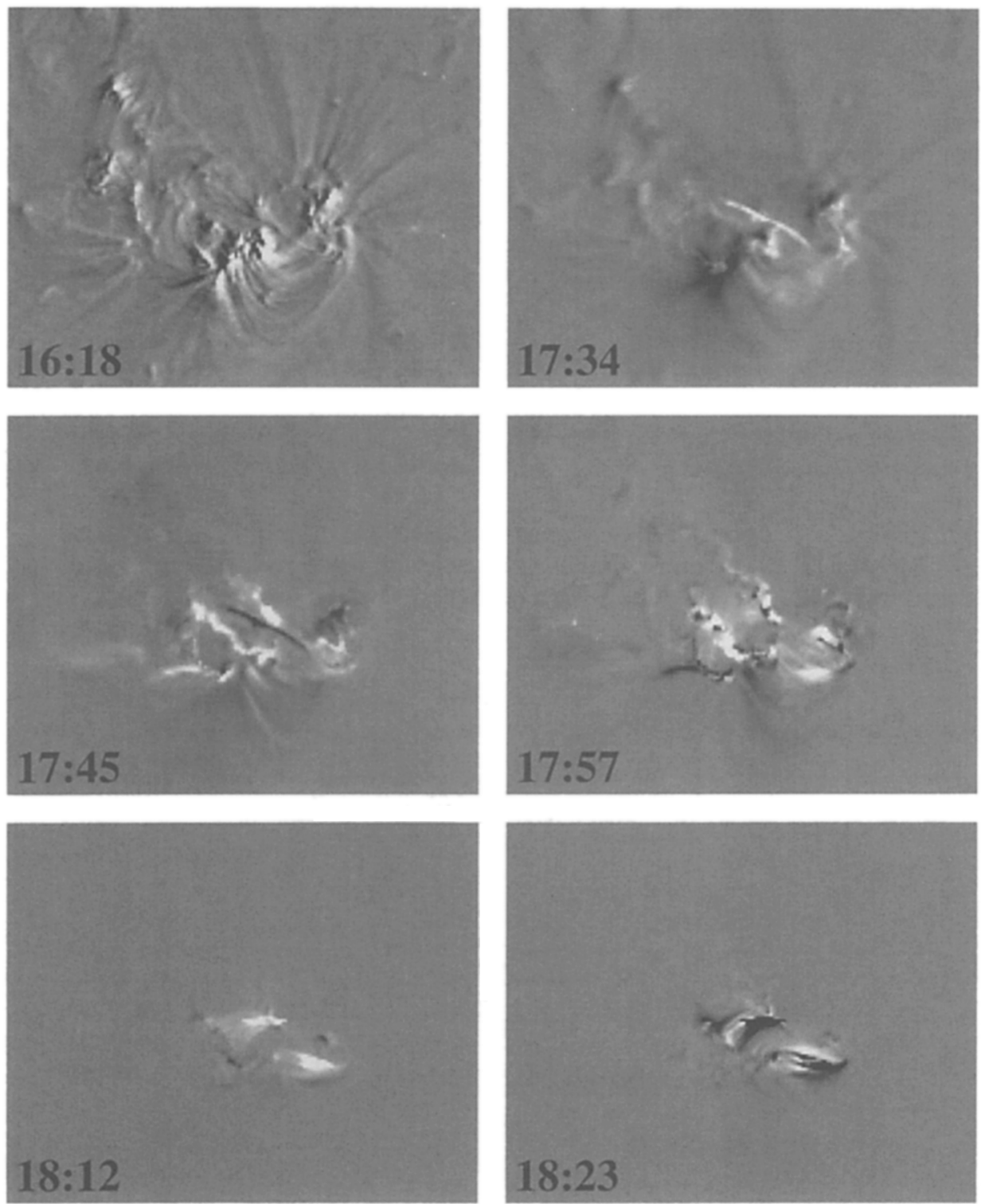

Figure 6. MDI/SOHO magnetogram of AR 8097 on 1997 October 21 at $1739 \mathrm{UT}$ 

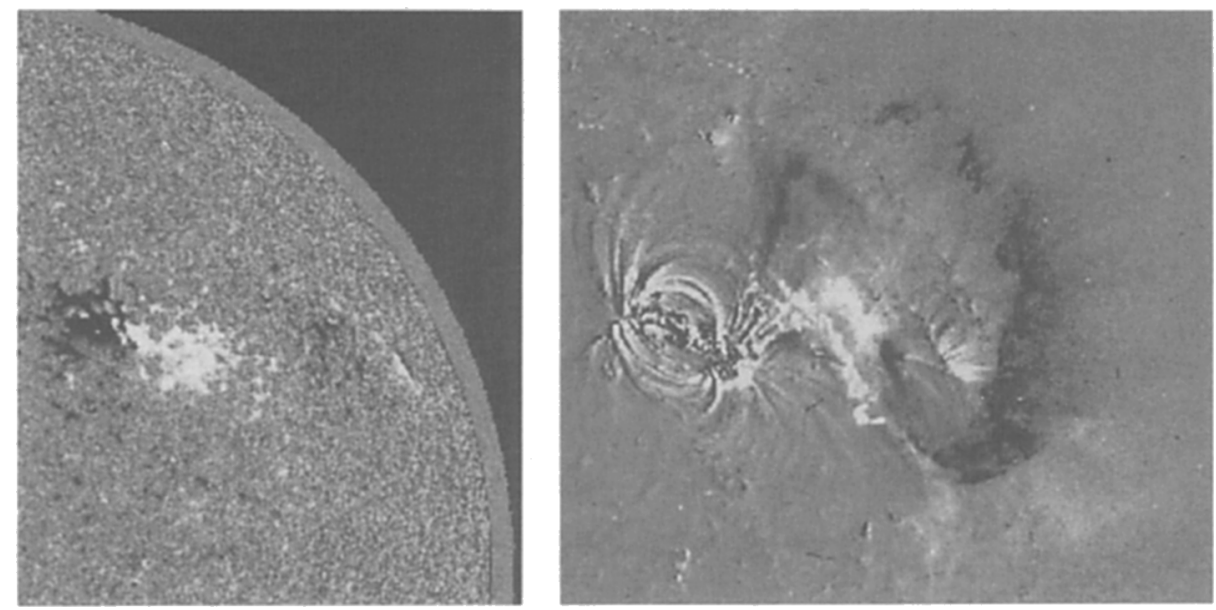

Figure 7. Left, MDI magnetogram at 0939 UT on 1998 May 19. Right, EIT $195 \AA$ running difference images of the eruptive prominence at $0956 \mathrm{UT}$. The two images are coaligned at their western (left) edge.
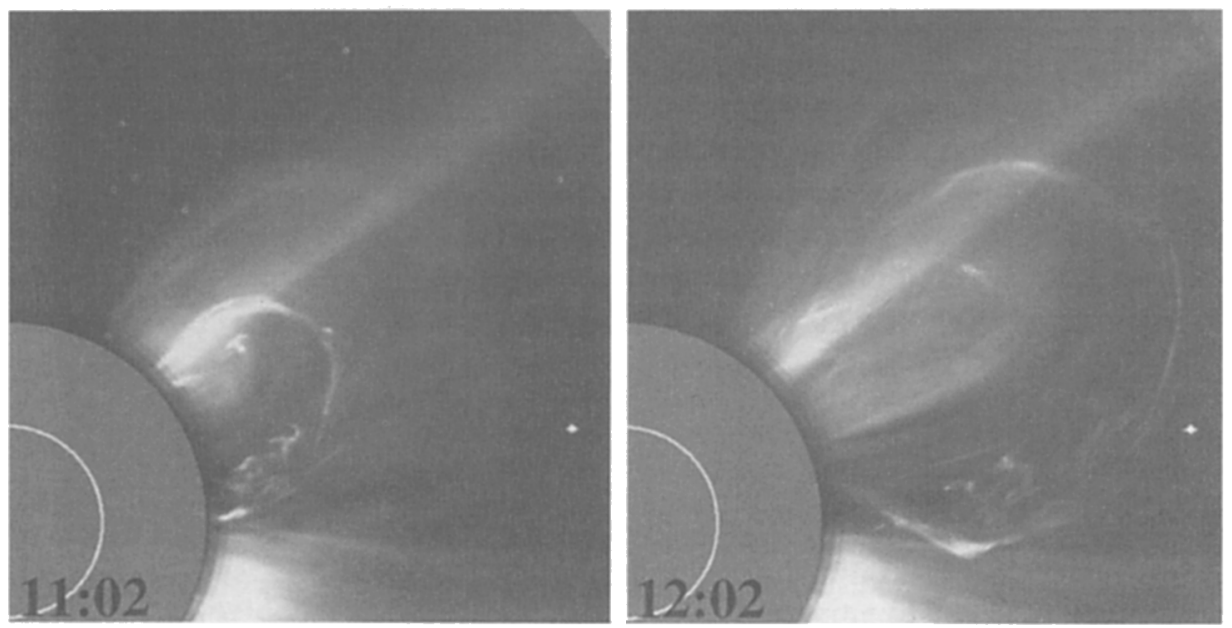

Figure 8. LASCO C2 images of the 19 May 1998 CME. The images are ratios to a background model. 


\section{Discussion}

We find that the set of CMEs that we have selected for being well observed on the solar disk tend to come from active regions with and without filaments and from quiescent filaments during the 1996-1998 period examined. Our examination of relationship of CMEs to the long term development of active region magnetic fields indicates that CMEs tend to come from the early and mid-life periods of active regions but CMEs are certainly also produced by decaying active regions. Increasing levels of small-scale magnetic flux emergence and cancellation tend to be correlated with the strength of CME production. The relationship of CMEs associated with the eruption of quiescent filaments to the evolution of the photospheric fields is difficult to determine.

Acknowledgments. This work was funded by NASA. SOHO is a project of international cooperation between ESA and NASA. The SOHO/LASCO data used in this paper are produced by a consortium of the Naval Research Laboratory, Max-Planck-Institut für Aeronomie (Germany), Laboratoire d'Astronomie (France), and the University of Birmingham (England). We gratefully acknowledge the SOHO MDI team for use of data from the MDI instrument. NSO/Kitt Peak data used here are produced cooperatively by NSF/NOAO, NASA/GSFC, and NOAA/SEL.

\section{References}

Canfield, R. C., Hudson, H. S., McKenzie, D. E. 1999, GRL, 26, 627

Dere, K. P., Brueckner, G. E., Howard, R. A., Michels, D. J., Delaboudiniere, J. P. 1999, ApJ, 516, 465

Falconer, D. A. 2000, GRL, in press

Feynman, J., and Martin, S. F. 1995, JGR, 100, 3355

Lara, A., Gopalswamy, N., and DeForest, C. 2000, GRL, 27, 1435

Luhmann, J. G., Gosling, J. T., Hoeksema, J. T., and Zhao, X. 1998, JGR, 103, 6585

Munro, R. H., Gosling, J. T., Hildner, E., MacQueen, R. M., Poland, A. I., and Ross, C. L. 1979, Solar Phys., 61, 201

St. Cyr, O. C., and Webb, D. F. 1991, Solar Phys., 136, 379

Webb, D. F., and Hundhausen, A. J. 1987, Solar Phys., 108, 383 